\title{
Design, Expression, and Structural Characterization of Hybrid Proteins of Samia cynthia ricini and Bombyx mori Silk Fibroins
}

\author{
Tetsuo AsaKura, ${ }^{\dagger}$ Hidetoshi KATO, Juming YAO, Raghuvansh Kishore, \\ and Makoto SHIRAI* \\ Department of Biotechnology, Tokyo University of Agriculture and Technology, Koganei, Tokyo 184-8588, Japan \\ ${ }^{*}$ Laboratory of Molecular Genetics, School of Agriculture, Ibaraki University, Ami, Ibaraki 300-0393, Japan
}

(Received August 21, 2002; Accepted October 18, 2002)

\begin{abstract}
Genetically engineering strategies have been applied to obtain the designed proteins [GGAGSGYGGGYGHGYGSDGG(GAGAGS $\left.)_{3}\right]_{n}$ of varying chain lengths $(n=2,4$ or 6); SLP2, SLP4, and SLP6, respectively. The amino acid sequence of the monomeric unit represents the incorporation of the consensus repeat sequences of the glycinerich region of Samia cynthia ricini followed by the repetitive crystalline region of Bombyx mori silk fibroins. The oligonucleotides were designed to encode the desired monomeric unit of silk-like hybrid proteins, which were then polymerised and expressed in Escherichi coli system. The monomer, SLP1, was synthesized chemically using solid phase method. The recombinant proteins were identified by N-terminal amino-acid sequencing and Western blotting. The structural characterizations of SLP1 and SLP6 samples were performed using ${ }^{13} \mathrm{C} \mathrm{CP} / \mathrm{MAS}$ NMR in the solid state. The structure of SLP1 in the solid state was distorted $\beta$-turn by judging from the chemical shift and line shape of the $\mathrm{C}^{\beta}$ carbon of Ala residue, and the structural transition from distorted $\beta$-turn to $\beta$-sheet occurred partially in SLP6. The solution structure was studied in hexafluoro-2-propanol (HFIP) using CD method. Although both samples, SLP1 and SLP6, showed indication of the ordered structures, marked change in the band intensities was observed between two CD spectra. Especially, the CD pattern of SLP6 is in agreement with the pattern with right-handed 310 -helical conformation.

KEY WORDS Silk-like Hybrid Proteins / Genetically Engineered Proteins / ${ }^{13} \mathrm{C}$ Cross Polarization Magic Angle Spinning NMR / Circular Dichroism / B. mori and S. c. ricini Silk Fibroins /
\end{abstract}

Natural silks have become the subject of extensive study as model systems for high-performance biopolymers and polymer composites in part because of their impressive mechanical properties of the fibers. ${ }^{1}$ In addition to high quality textiles, silks have been recognized to have not only excellent enzyme immobilization properties but also suggested to find potential applications in design and development of various biotechnological and biomedical devices. ${ }^{2-10}$

The unique amino acid composition and the primary structure of the silk fibroin appear to be important ${ }^{9}$ despite the fact that there are wide variations in the primary and secondary structures among different silks. For example, a well-known fibrous protein from the domesticated silkworm, B. mori, exhibits the amino acid composition (in mol\%) as $42.9 \%$ Gly, $30.0 \%$ Ala, $12.2 \%$ Ser, $4.8 \%$ Tyr, and $2.5 \%$ valine. ${ }^{9}$ On the other hand, the amino acid composition of the silk fibroin from a wild silkworm, S. c. ricini, is quite different. While the sum of Gly and Ala residues (82\%) is similar to B. mori $(73 \%)$ silk, the relative proportions of these residues are reversed (48.4\% Ala and 33.2\% Gly) in S. c. ricini. ${ }^{11,12}$ An analysis of the amino acid sequence of $B$. mori silk fibroin reported by Zhou et $a l .{ }^{13}$ revealed the presence of unusual highly repetitive GAGAGS sequence motifs, comprising about 55\% of the entire fibroin molecule whereas, the silk fibroin of S.c. ricini has been shown to consist of the relative much longer glycine-rich motifs: GGAGSGYGGGYGHGYGSDGG, also referred as amorphous region, flanked by poly-L-alanine (PLA) regions. ${ }^{12,14}$ The available information points towards that the (GAGAGS $)_{n}$ motifs may contribute significantly to the high strength of B. mori silk fiber. Extensive investigations of naturally produced silk materials have clearly indicated that there exists a strong correlation between the amino acid sequence and conformation-property relationships. Therefore, the production of high quality fibers with distinct mechanical properties would require not only the design of primary structure but also the precise knowledge of their secondary structure. The available information of the amino acid sequence motifs, i.e., the peptide modules, may be valuable. Moreover, the mixing of the modules in different proportions, by genetic engineering methods, would provide a unique opportunity to evaluate and produce the fibers with desired tensile strengths that may find potential medical and biotechnological applications. 
For native silk fibroin from $S$. c. ricini stored in the silkgland or the film prepared from the silk fibroin by drying it gently at room temperature, ${ }^{14,15}$ the PLA regions predominantly take an $\alpha$-helical structure. ${ }^{11,16-18}$ On the other hand, by stretching the film or immersing it into methanol-water mixture, the structure of PLA changes to $\beta$-sheet structures. However, only limited information are available on the glycine-rich regions of S. c. ricini. ${ }^{12,14}$ The two well-known crystalline forms of B. mori silk fibroins are the silk I (structure in the solid state before spinning) and silk II (structure in the solid state after spinning) forms, often referred as the two dimorphs of the fibroin molecules. Using a simple model peptide $(\mathrm{AG})_{15}$, the conformational characteristics of the 'less stable' silk I form have recently been clarified as a repeated type II $\beta$-turn structure (Ala; $\phi_{i+1} \sim-60^{\circ}, \psi_{i+1} \sim 130^{\circ}$, Gly; $\phi_{i+2} \sim 70^{\circ}, \psi_{i+2}$ $\sim 30^{\circ}$ ) stabilized by intramolecular hydrogen bonds. ${ }^{19}$ On the other hand, we used ${ }^{13} \mathrm{C}$ solid state NMR to clarify the heterogeneous structure of the natural fiber and the crystalline region from $B$. mori silk fibroin with silk II form. ${ }^{20,21}$ The relative proportions of the various heterogeneous components have been determined from their relative peak intensities. The various combinations of specific fibroin sequence motifs are expected to yield variations in the fiber's properties and may provide an opportunity to design unique biomaterials.

In this study, we attempted to synthesize the silklike hybrid proteins with defined sequence: [GGAGSGYGGGYGHGYGSDGG (GAGAGS) $\left.{ }_{3}\right]_{n}$, which have the primary structure incorporating the Gly-rich motif of $S$. c. ricini at the N-terminus and the highly repetitive motif (GAGAGS) $)_{n}$ of $B$. mori silk fibroin at the $\mathrm{C}$-terminus. If we can prepare the fiber from these silklike hybrid proteins, the fiber is expected to have different physical and mechanical properties from the original B. mori and S. c. ricini silk fibroin fibers. This provides us a variety of biomaterials with different properties. Thus, we can find out the biomaterials which are suitable to our individual purpose. In the process of the preparation, it might be possible to find out the fibers with both high strength and high toughness. The structural analyses of the monomeric and hexameric polypeptides, SLP1 and SLP6, respectively, in the solid state, were performed using ${ }^{13} \mathrm{C}$ cross polarization/magic angle spinning (CP/MAS) NMR. In addition, the circular dichroism (CD) measurement was made in the hexafluoro-2-propanol (HFIP) solvent system.

\section{EXPERIMENTAL}

\section{Materials}

E. coli strain $\mathrm{DH} 5 \alpha$ was used for propagation and construction of plasmids, and E. coli strain BL21(DE3)pLysS was used for production of proteins. Synthesized DNA fragments with phosphorylation were purchased from Sigma Genosys, Japan. Restriction enzymes and ligase were purchased from Takara Shuzo, Japan. Plasmid pUC118 was obtained from Takara Shuzo, and pET30a was obtained from Novagen. All other chemicals were of analytical grade. Bacterial growth in rich medium, DNA manipulations, and transformation conditions were performed as described in the reference by Sambrook et al. ${ }^{22}$ DNA sequencing was performed on a ABI PRISM ${ }^{\mathrm{TM}} 377$ Auto Sequencer according to its user's manual. Cell density was measured on a Hitachi U-3200 spectrophotometer in quartz cuvettes with a path length of $1 \mathrm{~cm}$ at $\lambda=$ $600 \mathrm{~nm}$. Batch cultures were performed on MDL-6C Fermentor (B. E. Marubishi Co.), with $1.2 \mathrm{~L}$ working volume.

\section{Construction of pUC118-linker}

Figure 1a shows a synthetic adapter containing $X b a$ I, Spe I, and Nhe I restriction enzyme sites, which was ligated into pUC118 that had been digested with $X b a$ I and dephosphorylated using Calf Intestine Alkali Phosphatase (CIAP), to form the ampicillin resistant pUC118-linker. The ligation mixture was used to transform $E$. coli strain DH5 $\alpha$ with selection on $2 \times \mathrm{YT}$ agar medium containing $50 \mu \mathrm{g} \mathrm{mL}^{-1}$ ampicillin. Recombinant plasmid pUC118-linker was examined by doublestranded DNA sequencing with M13 primers M4 and $\mathrm{RV}$. The sequencing reaction was performed according to standard PE Biosystems sequencing protocol.

\section{Construction of Monomer Fragment}

Four oligonucleotides shown in Figure $1 \mathrm{~b}$ were annealed to generate two DNA monomer fragments encoding $S$. c. ricini Gly-rich and B. mori crystal regions, respectively. The purified duplex DNAs containing the Bam H I cleavage sites at both fragments were ligated into the BamH I site of pUC118. The transformation and sequencing were performed in the same manner as described above. After digestion of the recombinant pUC118 containing a monomer fragment with Nhe I and Spe I, the fragment was ligated into Nhe I-Spe I site of pUC118-linker. 


\section{a. linker \\ Xbal Spel Nhel Xbal \\ 5' CT AGA ATG ACT AGT GGG CCC GCT AGC ATG T 3 ' \\ 3' T TAC TGA TCA CCC GGG CGA TCG TAC AGA TC 5 \\ Met Thr Ser Gly Pro Ala Ser Met}

\section{b. S.c.riciniGly-rich region}

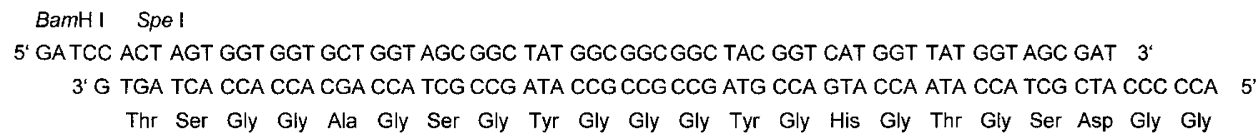

Figure 1. The designed oligonucleotide sequences for silk-like proteins (SLPs). a :DNA sequence of synthetic adapter inserted into pUC118 to create pUC118-linker and b :oligonucleotide sequences used for cloning of the hybrid proteins SLP2, SLP4, and SLP6.

Table I. Construction and over-expression of the multimer hybrid proteins with $\mathrm{pET} 30$ a expression system

\begin{tabular}{clc}
\hline Multimer & \multicolumn{1}{c}{ Combination strategy } & Yield $\left(\mathrm{mg} \mathrm{L}^{-1}\right)$ \\
\hline SLP 2 & Monomer DNA fragment + linear vector containing monomer DNA & 21.0 \\
SLP 4 & Dimer DNA fragment + linear vector containing dimer DNA & 38.7 \\
SLP 6 & Dimer DNA fragment + linear vector containing tetramer DNA & 31.0 \\
\hline
\end{tabular}

\section{Construction of Multimers}

To construct the multimers, a plasmid pUC118 containing the monomer (or multimers) was digested with Nhe $\mathrm{I},{ }^{23}$ generating a linearized vector containing insert. A second insert-containing plasmid was digested with Nhe I and Spe I, liberating the insert DNA fragment. Linearized vectors and insert DNA fragments were extracted from the agarose slice using low-speed centrifugation and ligated in different combinations to provide higher order multimers. Recombinant multimers are listed in Table I, along with the strategy used in each construction. All of these DNA multimers were released from pUC118 and then constructed into pUC118-linker. After digestion of the recombinant pUC118-linker containing multimerized DNA fragments with BamH I and Hind III, the target DNAs encoding the hybrid proteins were extracted from the agarose gel and inserted into the BamH I-Hind III sites of pET30a, generating pET30a-SLP series. The ligation mixture was used to transform a host strain BL21(DE3)pLysS.

\section{Protein Expression and Purification}

Cultures were grown at $37^{\circ} \mathrm{C}$ in $2 \times \mathrm{YT}$ medium with chloramphenicol $\left(25 \mu \mathrm{mL}^{-1}\right)$ and kanamycin $\left(25 \mu \mathrm{mL}^{-1}\right)$ to an $\mathrm{OD}_{600}=0.5-1.0$, and the expression of hybrid proteins were induced by the addition of 1 mM IPTG. After $1-3 \mathrm{~h}, 1 \mathrm{~mL}$ of cell culture was har- vested and the cells were lysed in $1 \mathrm{~mL}$ of $2 \times$ sample buffer. Lysates were resolved by SDS-PAGE using $10-12 \%$ polyacrylamide gel. For large-scale expression, cultures were grown in $1.2 \mathrm{~L}$ of TB medium by using fermentor to $\mathrm{OD}_{600}=1.0$, IPTG was added to $1 \mathrm{mM}$. After $4 \mathrm{~h}$ the cells were harvested by centrifugation. Recombinant proteins were purified by affinity chromatography on a Ni-NTA column in accordance with the protocol of manufacturer. Recombinant protein containing $6 \times$ His was monitored by Western blotting using a $6 \times$ His antibody and HRP conjugated rabbit IgG. Purified proteins were exhaustively dialyzed against distilled water and then lyophilised to provide solid materials.

\section{Cleavage of Hybrid Proteins with Cyanogen Bromide}

The cyanogen bromide cleavage of the hybrid proteins was performed according to the procedure described by Fukushima et al. ${ }^{24}$ Approximately $40 \mathrm{mg}$ of purified hybrid His $_{6}$-SLP protein was dissolved in $10 \mathrm{~mL}$ of $99 \%$ formic acid and diluted to $70 \%$ formic acid with distilled water. The mixture by added $100 \mathrm{mg}$ of $\mathrm{CNBr}$ crystals was stirred at room temperature for $24 \mathrm{~h}$ and transferred into a dialysis membrane with a molecular weight cutoff of 3500 . The solution was dialyzed against distilled water for $3 \mathrm{~d}$, and the cleaved protein $(21 \mathrm{mg})$ was recovered by lyophilization. 


\section{Peptide Synthesis}

The monomeric unit [GGAGSGYGGGYGHGYGSDGG(GAGAGS) $)_{3}$ AS] was synthesized by solid phase F-moc chemistry on a fully automated Pioneer Peptide Synthesis System (Applied Biosystems Ltd.) as described elsewhere. ${ }^{19,21}$ The peptide was assembled on F-moc-Gly-PEG-PS resin and the coupling of F-moc amino acids were performed. After synthesis, the free peptides were released from the resin by the treatment with a $40 \mathrm{~mL}$ mixture of TFA, phenol, triisopropylsilane and water (88:5:2:5\% by volume) for two hours at room temperature. The crude peptide was precipitated, washed with cold diethyl ether and dried under vacuum. The precipitate was dissolved in $9 \mathrm{M} \mathrm{LiBr}$ and dialyzed extensively against distilled water. The peptide was collected by lyophilization.

\section{Cp Fraction of B. mori Silk Fibroin}

The $\mathrm{Cp}$ fraction of $B$. mori silk fibroin was prepared from regenerated silk fibroin solution as described elsewhere. ${ }^{21}$ Chymotrypsin (40 mg, Seikagaku Kogyo Co., Japan), dissolved in a few $\mathrm{mL}$ of water, was added to an aqueous solution of about $4 \mathrm{~g}$ of fibroin buffered with $\mathrm{Na}_{2} \mathrm{HPO}_{4} \cdot 12 \mathrm{H}_{2} \mathrm{O}$ and $\mathrm{NaH}_{2} \mathrm{PO}_{4} \cdot 2 \mathrm{H}_{2} \mathrm{O}$ at $\mathrm{pH}$ 7.8. The solution $(200 \mathrm{~mL})$ was incubated at $40^{\circ} \mathrm{C}$ for $24 \mathrm{~h}$, and the precipitate that formed (Cp fraction) was separated by centrifuging at $10000 \mathrm{rpm}$ followed by washing with $0.03 \mathrm{~N} \mathrm{HCl}$ to inactivate the enzyme reaction. Then the precipitate was washed several times with distilled water, ethyl alcohol, and ethyl ether. Finally the precipitate was dried in vacuum, yielding $56 \%$ of original fibroin. The structure of this Cp fraction is silk II. The $\mathrm{Cp}$ fraction in the silk I form was prepared by dialyzing the $\mathrm{Cp}$ fraction in $60 \%$ aqueous $\mathrm{LiBr}$ against similar solutions of $\mathrm{LiBr}$ that were diluted progressively with water. This procedure results in a precipitate with the silk I form.

\section{Solid State NMR Observation}

Solid state ${ }^{13} \mathrm{C} C P / M A S$ NMR spectra were acquired on a Chemagnetics CMX-400 spectrometer operating at $100 \mathrm{MHz}$, with a CP contact time of $1 \mathrm{~ms}$, TPPM decoupling, and magic angle spinning at $10 \mathrm{kHz}$. A total of 10000-25000 scans were collected over a spectral width of $60 \mathrm{kHz}$, with a recycle delay of $3 \mathrm{~s}$. Chemical shifts are reported relative to TMS as reference.

\section{Circular Dichroism}

The solution-state CD spectra (185-260 nm) of SLP1 and SLP6 were recorded in HFIP (Wako Pure Chemical Industries Ltd.) on a JASCO J-805 Spectropolarimeter. A cylindrical quartz cell of $0.01 \mathrm{~cm}$ was used for spec- tral measurements. The spectra were recorded at an ambient temperature and each spectrum is presented as an average of eight consecutive scans measured at $1 \mathrm{~nm}$ resolution. The data were processed on a computer with the available software and results are presented as residual molar ellipticity $[\theta]_{M}\left(\mathrm{deg} . \mathrm{cm}^{2} \mathrm{dmol}^{-1}\right)$ based on number of residues 38 for monomer and 228 for hexamer, with the molecular weights 2889 and 17334, respectively.

\section{RESULTS AND DISCUSSION}

\section{Construction of Monomeric and Multimeric SLP DNAS}

In order to produce the tandem repetitive polypeptides, DNA sequences shown in Figure $1 \mathrm{~b}$ were designed: The 5'-Bam $\mathrm{H}$ I site at $S$. c. ricini Gly-rich region and 3'-BamH I site at $B$. mori crystalline region are positioned at each end for insertion of the DNA into BamH I site of cloning plasmid pUC118. The Spe I and Nhe I restriction sites encoding Thr-Ser and AlaSer were inserted after and before Bam $\mathrm{H}$ I sites, respectively, in order to isolate the DNA fragments from the recombinant plasmids. Optimal codons for each amino acid can be selected using codon usage in E. coli. ${ }^{24}$ Since the polypeptide expression level and the stability of the DNA itself are influenced by the base composition and sequence of the DNA, the codons were selected as many as A-T contents as possible. The DNA multiers were inserted into Nhe I and Spe I digested pUC118-linker, flanked by an ATG codon encoding methionine at each site in order to obtain the periodic portion of SLP by cleavage of the expressed hybrid proteins with cyanogens bromide (CNBr).

Plasmids, pUC118 containing tandem repeats of the synthetic DNA fragments were identified by restriction digestion analysis using BamH I and DNA sequencing. Multimers were formed by insertion of Nhe I-Spe I fragments into Nhe I digested vectors since, these enzymes generate identical cohesive ends, to build, in a tightly controlled fashion, larger genes of any desired degree of multimerization. We have exploited these strategies for the controlled construction of multimers ranging in size from 1 to 6 repeats of the SLP sequence while retaining unique Nhe I and Spe I sites in the constructs. The constructs of SLP multimers are summarized in Table I. The agarose gel electrophoresis of these constructs after $B a m \mathrm{H}$ I digestion are shown in Figure 2 and the results are the same as our expected base pairs.

\section{Protein Expression and Analysis}

Our efforts for expression utilized the commercially available expression vector pET30a which places the 


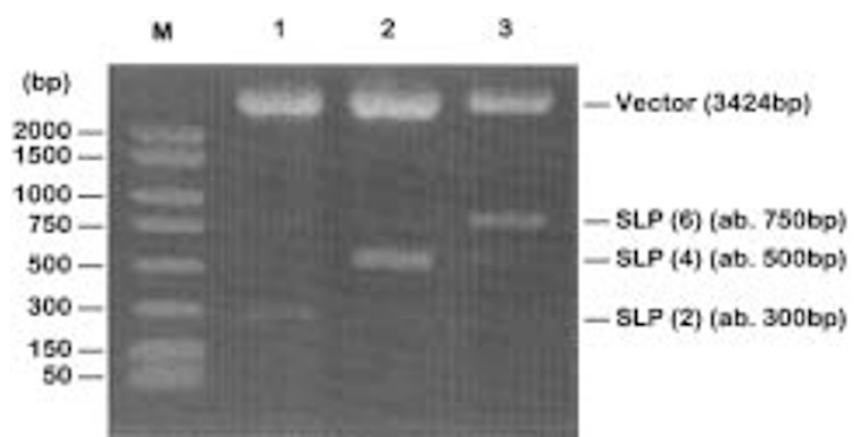

Figure 2. Bam H I digestion analysis of multimerized oligonucleotide sequences. M: molecular weight markers; lane 1: pUC118-SLP2; lane 2: pUC118-SLP4 and lane 3: pUC118-SLP6.

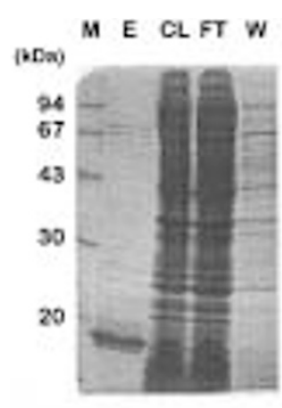

a

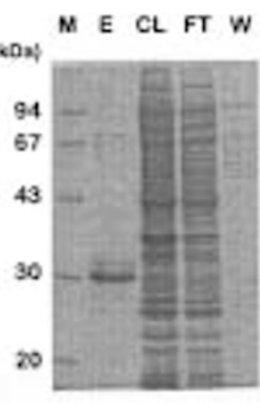

b

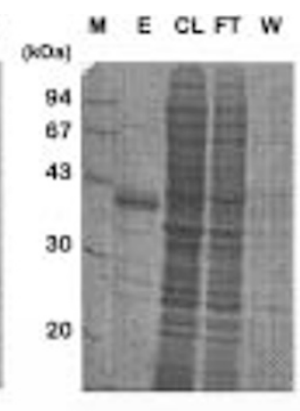

$\circ$
Figure 3. Identification of the recombinant hybrid proteins $\mathrm{His}_{6}$-SLP2, $\mathrm{His}_{6}$-SLP4, and $\mathrm{His}_{6}$-SLP6 obtained at various stages of purifications. a :hybrid protein $\mathrm{His}_{6}-\mathrm{SLP} 2$ produced using pET30a-SLP2 and after Ni-NTA column chromatography, b : hybrid protein $\mathrm{His}_{6}$-SLP4 produced using pET30a-SLP4 and $\mathbf{c}:$ hybrid protein $\mathrm{His}_{6}$-SLP6 produced using pET30a-SLP6. The protein bands were visualized by Coomassie staining. M: molecular weight markers; E: after elution with $250 \mathrm{mM}$ imidazole solution, CL: cell lysate, FT : flow-through, and W: after washing with $20 \mathrm{mM}$ imidazole solution. The hybrid proteins were eluted with $250 \mathrm{mM}$ imidazole solution, which have the molecular weight of $19 \mathrm{kDa}(\mathbf{a E})$, $29 \mathrm{kDa}(\mathbf{b E})$, and $40 \mathrm{kDa}(\mathbf{c E})$, respectively.

synthetic genes under the control of the bacteriophage T7 promoter and places a unique $6 \times$ His sequence at the N-terminus of the recombinant protein for purification by immobilized metal affinity chromatography. pET30a-SLPs were transformed into E. coli BL21(DE3)pLysS and the encoded proteins were expressed upon IPTG induction. Crude extracts were analysed by polyacrylamide gel electrophoresis and Western Blotting using an anti-His antibody. Staining of the gels with Coomassie Blue showed clear bands of $\mathrm{His}_{6}$-SLP2, $\mathrm{His}_{6}$-SLP4, and His 6 -SLP6 proteins, which have the molecular weight of $19 \mathrm{kDa}, 29 \mathrm{kDa}$, and $40 \mathrm{kDa}$, respectively (Figure 3). However, higher order multimers (His 6 -SLP8 and His 6 -SLP12) which were readily detected by Western Blotting analysis, did not appear as visible bands (data not shown). This has been observed in the expression of other silk-like recombinant proteins. ${ }^{25,26}$ The antibody used in the Western

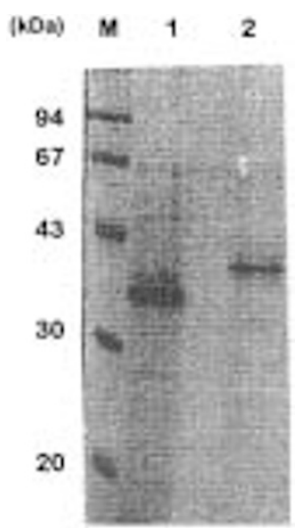

Figure 4. SDS-PAGE of purified hybrid proteins produced from pET30a-SLP6. M: molecular weight marker; Lane 1: CNBrcleaved protein, SLP6; Lane 2: uncleaved protein His 6 -SLP6.

Blotting assays was not quantitative in its reaction with silk-like proteins but could be reliably used to detect synthetic hybrid proteins both in whole cell lysates and in purified form. In conjunction with the physical characterization of the purified materials (vide infra), the data support the expression of SLP multimers using the pET expression system.

Proteins expressed from plasmid pET30a-SLP were purified on a Ni-NTA column through the $6 \times$ His sequence positioned at the $\mathrm{N}$-terminus. We focused our characterization efforts on three distinct size variants of the SLP multimer sequences. Multimers containing 2, 4, and 6 repeats of the 38 amino acids, that is, SLP2, SLP4, and SLP6, respectively, were examined. Typical yields for the dried proteins were 21.0, 38.7, and $31.0 \mathrm{mg} \mathrm{L}^{-1}$ of medium culture, respectively, (Table I). These proteins were further purified by $\mathrm{CNBr}$ cleavage and then by dialysis. Figure 4 compares the SDSPAGE results of the $\mathrm{His}_{6}$-SLP6 (lane 2) and the CNBrcleaved protein, SLP6 (lane 1). The cleaved product was observed as a single predominant band and uncleaved protein was not remained under the cleavage conditions, suggesting that the purity of this product is high. The molecular weight of the cleaved protein is around $36 \mathrm{kDa}$. All of the proteins were identified by $\mathrm{N}$-terminal amino acid sequencing (data not shown).

\section{Solid State ${ }^{13}$ C NMR Analysis}

The solid-state ${ }^{13} \mathrm{C}$ NMR chemical shifts of polypeptides and proteins have been reported to be extremely sensitive to backbone conformation of the constituent residues and have been exploited for the structural analysis of silk fibroin. ${ }^{27}$ Figure 5 compares the ${ }^{13} \mathrm{C}$ CP/MAS solid state NMR spectra of SLP1 (a) and SLP6 (b). As for comparison, the spectra of Cp fraction in silk I and silk II forms are also shown in Figure 5c and $5 \mathrm{~d}$, respectively. The $\mathrm{Cp}$ fraction predominantly 


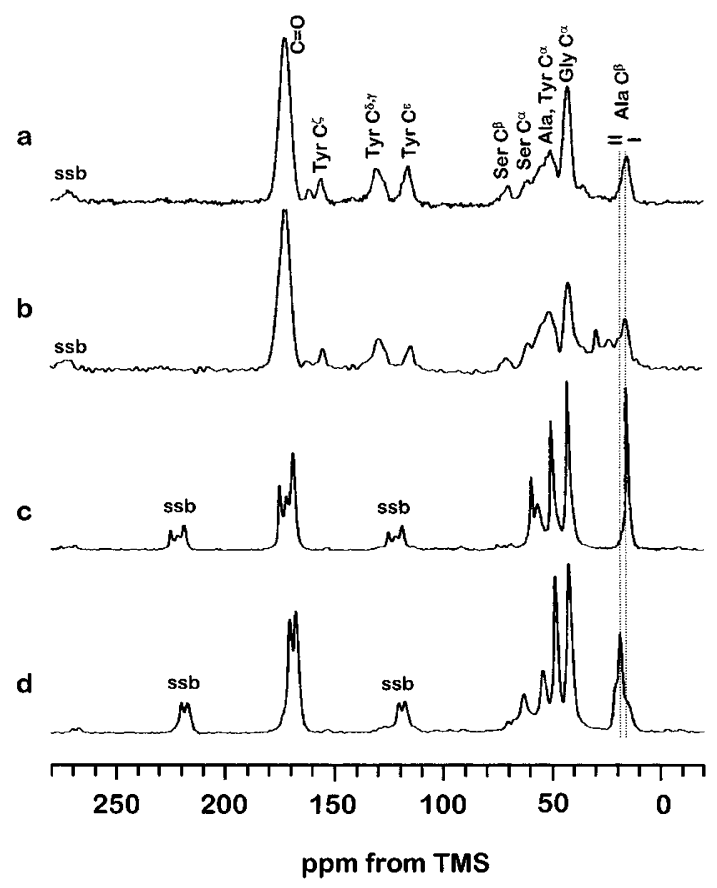

Figure 5. ${ }^{13} \mathrm{C} \mathrm{CP} / \mathrm{MAS} N \mathrm{MR}$ spectra. a: monomeric peptide, SLP1; b: hybrid protein, SLP6; $\mathbf{c}$ : Cp fraction in silk I form; d: Cp fraction in silk II form. The conformational assignments of the Ala $\mathrm{C}^{\beta}$ peak, I (Silk I) and II (Silk II), are indicated by the dotted lines in the spectra.

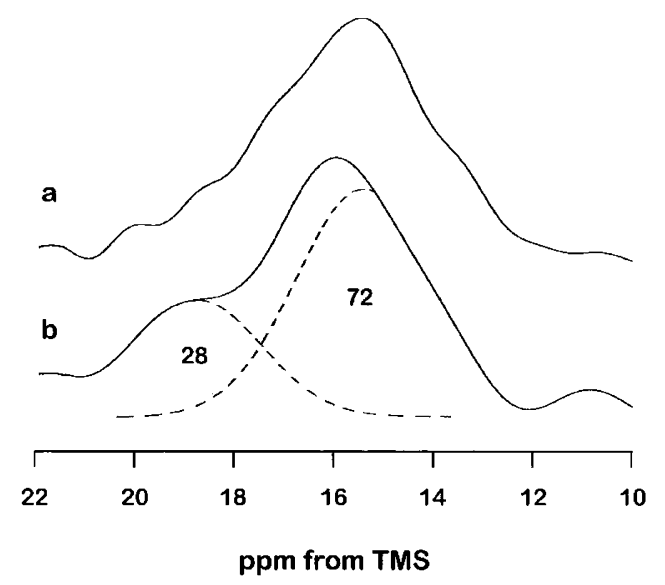

Figure 6. Expanded Ala $\mathrm{C}^{\beta}$ peaks of ${ }^{13} \mathrm{C} \mathrm{CP} / \mathrm{MAS}$ NMR spectra of (a) monomeric peptide, SLP1 and (b) hybrid protein, SLP6. The spectral deconvolution was performed by assuming Gaussian.

has the amino acid sequence of (GAGAGS) ${ }_{n},{ }^{9,13}$ The resonance assignments of the constituent amino acids are identified from their characteristic peak positions and by comparing with reported chemical shift data of the silk fibroins and their derived peptides. ${ }^{27}$ As judged from the characteristic chemical shift values of the Ala and Gly residues (Ala $C^{\alpha} \sim 50.5$, Ala $C^{\beta} \sim 15.9$, Gly $\mathrm{C}^{\alpha} \sim 42.5 \mathrm{ppm}$ ) (Figure 5a), it might be concluded that the SLP1 takes silk I structure. However, the broad lineshape of Ala $C^{\beta}$ peak (Figures 5a and 6a) suggests that it takes a distorted $\beta$-turn. ${ }^{20,21}$ On the other hand, Figure $6 \mathrm{~b}$ shows that the chemical shift of Ala $C^{\beta}$ peak

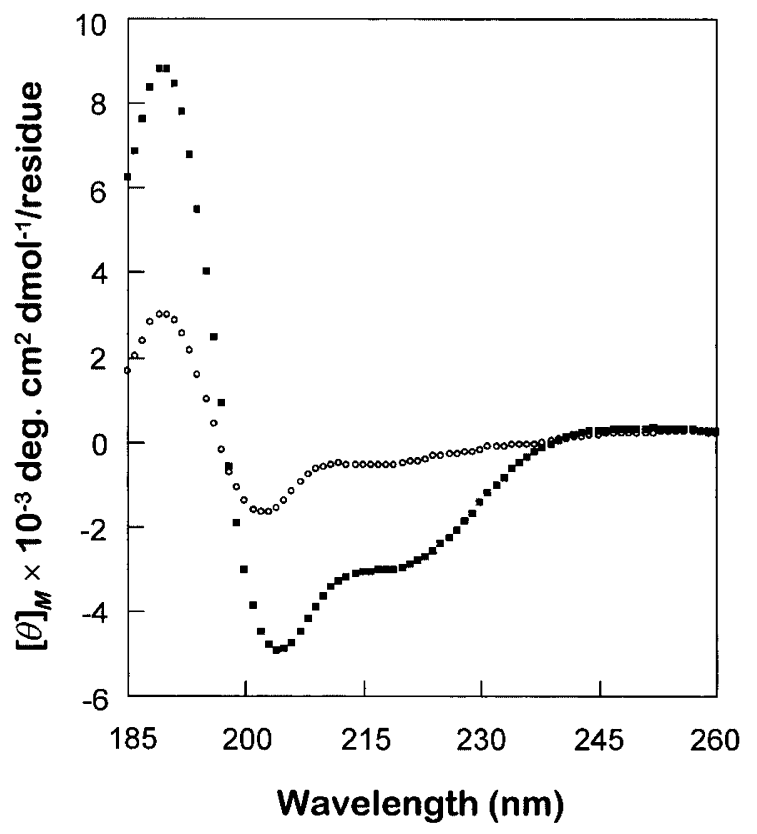

Figure 7. A comparison of the CD spectra of SLP1 $(\bigcirc)$ and SLP6 (ם) in HFIP solution. The characteristic band positions and the residual molar ellipticity values are described in the text.

of SLP6, as well as SLP2 and SLP4 (data not shown) is significantly different from that of SLP1 although the difference is small. Namely, the presence of small amounts of $\beta$-sheet structure is expected in SLP6 sample. The Ala $C^{\beta}$ peak was divided into two peaks after careful peak simulation. The fraction was estimated to be $72 \%$ distorted $\beta$-turn and $28 \% \beta$-sheet. ${ }^{20,21}$

\section{Analysis}

In order to prepare silk fiber from the hybrid proteins, HFIP was used as the solvent and methanol was used as the coagulant for artificial spinning. ${ }^{28,29}$ However, we could not obtain the fiber. So we tried to characterize the solution structure of SLP1 and SLP6 by the $\mathrm{CD}$ method in HFIP solution. The analysis may provide an opportunity to directly correlate the secondary structural features of the silk derived peptides in the specific hydrophobic environment.

Figure 7 compares the CD spectra of SLP1 and SLP6 in HFIP. The observed CD spectrum of SLP1 is characterized by the presence of two weak negative bands: a shoulder at $\sim 223 \mathrm{~nm}\left([\theta]_{M} \sim-650 \mathrm{deg}\right.$. $\mathrm{cm}^{2} \mathrm{~mol}^{-1} /$ residue) and a relatively sharp band at $\sim 202 \mathrm{~nm}\left([\theta]_{M} \sim-1680 \mathrm{deg} . \mathrm{cm}^{2} \mathrm{~mol}^{-1} /\right.$ residue $)$ and an intense positive extremum at $\sim 190 \mathrm{~nm}\left([\theta]_{M} \sim\right.$ -2990 deg. $\mathrm{cm}^{2} \mathrm{~mol}^{-1} /$ residue). The crossover exists at $\sim 197 \mathrm{~nm}$. Interestingly, these spectral features in case of SLP6 are dramatically pronounced however, without any change in the band positions, i.e., a distinct negative shoulder $\sim 223 \mathrm{~nm}\left([\theta]_{M} \sim-2720 \mathrm{deg}\right.$. $\mathrm{cm}^{2} \mathrm{~mol}^{-1} /$ residue), an fairly strong band at $\sim 204 \mathrm{~nm}$ 
$\left([\theta]_{M} \sim-4950\right.$ deg. $\mathrm{cm}^{2} \mathrm{~mol}^{-1} /$ residue $)$ and a strong positive peak at $\sim 190 \mathrm{~nm}\left([\theta]_{M} \sim-8800\right.$ deg. $\mathrm{cm}^{2}$ $\mathrm{mol}^{-1} /$ residue). While the $\mathrm{CD}$ pattern of the monomer unit exhibits a clear inclination for the helical structure, the observed spectra of SLP6 clearly indicate the predominance of helical conformations stabilized by intra-molecularly hydrogen bond. Marked changes in the band intensities of the hexameric unit clearly suggest the existence of cooperative effects in the induction of secondary structures. On the contrary, based on CD studies of elastin-like peptides (VPGVG) ${ }_{n}$ (where $n$ varies between 1 to 5 ), the lack of co-operative effects has been suggested. ${ }^{30}$ Moreover, the 'cooperative unit' did not seem to exceed more than one repeat. Under the present experimental conditions, the results largely exclude the presence of $\beta$-sheet structures in solution condition. Of the two well-known, closely related helical secondary structures, i. e., $\alpha$ - and $3_{10}$-helices, the observed CD spectra in HFIP appear to be more close to a 310 -helical structure however, the presence of equilibrium between the two helical forms cannot be ruled out. It must be noted that the backbone torsion angles $\left(\phi \sim-50 \pm 20^{\circ}\right.$ and $\left.\psi \sim-30 \pm 20^{\circ}\right)$ characterize both the helical forms and subtle changes in the torsion angles may easily inter-convert the two structures. Interestingly, Toniolo and co-workers indicated the possibility of distinguishing the two helical forms from the experimental CD results from the characteristic ratio $R=[\theta]_{222} /[\theta]_{208} \cdot{ }^{31,32}$ For an ideal $3{ }_{10}$-helical structures, the characteristic ratio $R=[\theta]_{222} /[\theta]_{204}$, is suggested to be 0.4 whereas, it is close to 1.0 in the case of $\alpha$-helix. The CD results on the hexameric unit are more in favour of the polypeptides with right-handed $3{ }_{10}$-helical conformation, as judged from the relative intensities of the bands at 204 and $222 \mathrm{~nm}$.

\section{CONCLUSIONS}

We could not obtain silk fiber from the hybrid proteins with spinning method from the solution; HFIP as the solvent and methanol as the coagulant. However, SLP6 with the longest peptide sequence among the hybrid samples prepared here takes $28 \% \beta$-sheet structure in the solid state and takes ordered structure like $3_{10}$ helix in HFIP. Therefore the hybrid silk protein with longer sequence will be possible to prepare the fiber. The present study clearly indicates that combining the two structurally different characteristics from two or more different species may be an attractive possibility for exploring and comparing the functional properties of the silk materials. It appears encouraging that the present protocols for obtaining the hybrid SLPs in sufficient quantity, may further be extended to other related systems.

Acknowledgment. TA acknowledges Prof. D. Kaplan at Tufts University for stimulated discussion. TA also acknowledges support from the Program for Promotion of Basic Research Activities for Innovative Biosciences, Japan. The work was carried out partly under a JSPS Invitation Fellowship offered to R.K.

\section{REFERENCES}

1. H. Heslot, Biochimie, 80, 19 (1998).

2. A. Kuzuhara, T. Asakura, R. Tomoda, and T. Matsunaga, J. Biotechnol., 5, 199 (1987).

3. M. Demura, T. Asakura, and T. Kuroo, Biosensors, 4, 361 (1989).

4. M. Demura, T. Asakura, E. Nakamura, and H. Tamura, J. Biotechnol., 10, 113 (1989).

5. M. Demura and T. Asakura, Biotechnol. Bioeng., 33, 598 (1989).

6. T. Asakura, H. Yoshimizu, and M. Kakizaki, Biotechnol. Bioeng., 35, 511 (1990).

7. M. Demura, T. Komura, and T. Asakura, Bioelectrochem. Bioenerg., 26, 167 (1991).

8. M. Demura and T. Asakura, J. Membr. Sci., 59, 39 (1991).

9. T. Asakura and D. L. Kaplan, in "Encyclopedia of Agricultural Science", C. J. Arutzen and E. M. Ritter, Ed., Academic Press, Inc., New York, N.Y., 1994, vol. 4, pp 1-11.

10. Y. Liu, J. Qian, H. Liu, X. Zhang, J. Deng, and T. Yu, J. Appl. Polym. Sci., 61, 641 (1996).

11. T. Asakura and T. Murakami, Macromolecules, 18, 2614 (1985).

12. Y. Nakazawa and T. Asakura, FEBS Lett., 529, 188 (2002).

13. C. Z. Zhou, F. Confalonieri, N. Medina, Y. Zivanovic, C. Esnault, T. Yang, M. Jacquet, J. Janin, M. Duguet, R. Perasso, and Z. G. Li, Nucleic Acids Research, 28, 2413 (2000).

14. T. Asakura, T. Ito, M. Okudaira, and T. Kameda, Macromolecules, 32, 4940 (1999).

15. J. D. van Beek, L. Beaulieu, H. Schafer, M. Demura, T. Asakura, and B. H. Meier, Nature, 405, 1077 (2000).

16. T. Asakura, H. Kashiba, and H. Yoshimizu, Macromolecules, 21, 644 (1988).

17. Y. Nakazawa and T. Asakura, Macromolecules, 35, 2393 (2002).

18. T. Asakura, H. Yoshimizu, and F. Yoshizawa, Macromolecules, 21, 2038 (1988).

19. T. Asakura, J. Ashida, T. Yamane, T. Kameda, Y. Nakazawa, K. Ohgo, and K. Komatsu, J. Mol. Biol., 306, 291 (2001).

20. T. Asakura, J. Yao, T. Yamane, K. Umemura, and A. S. Ulrich, J. Am. Chem. Soc., 124, 8794 (2002).

21. T. Asakura and J. Yao, Protein Sci., 11, 2706 (2002).

22. J. Sambrook, E. F. Frisch, and T. Maniatis, Ed., "Molecular Cloning, A Laboratory Manual", 2nd ed, Cold Spring Harbor Laboratory Press, Cold Spring Harbor, NY, 1989.

23. T. Kempe, S. B. H. Kent, F. Chow, S. M. Peterson, W. I. Sundquist, J. L. Italien, D. Harbrecht, D. Plunkett, and W. J. DeLorbe, Gene, 39, 239 (1985).

24. Y. Fukushima, Biopolymers, 45, 269 (1998). 
25. J. Cappello, J. Crissman, M. Dorman, M. Mikolajczak, M. M. Textor, and F. Ferrari, Biotechnol. Prog., 6, 198 (1990).

26. K. P. McGrath, M. J. Fournier, T. L. Mason, and D. A. Tirrell, J. Am. Chem. Soc., 114, 727 (1992).

27. T. Asakura, M. Iwadate, M. Demura, and M. P. Williamson, Int. J. Biol. Macromol., 24, 167 (1999).

28. J. Yao, H. Masuda, C. Zhao, and T. Asakura, Macromolecules, 35, 6 (2002).
29. C. Zhao, J. Yao, H. Masuda, R. Kishore, and T. Asakura, Biopolymers, in press.

30. H. Reiersen, A. R. Clarke, and A. R. Rees, J. Mol. Biol., 283, 255 (1998).

31. C. Toniolo, A. Polese, F. Formaggio, M. Crisma, and J. Kamphuis, J. Am. Chem. Soc., 118, 2744 (1996).

32. M. Manning and R. W. Woody, Biopolymers, 31, 569 (1991). 